\title{
Ergodic Capacity Upper Bound for Dual MIMO Ricean Systems: Simplified Derivation and Asymptotic Tightness
}

\author{
Michail Matthaiou, Member, IEEE, Yannis Kopsinis, Member, IEEE, \\ David I. Laurenson, Member, IEEE, and Akbar M. Sayeed, Senior Member, IEEE
}

\begin{abstract}
An analytical upper bound on the ergodic capacity of Multiple-Input Multiple-Output (MIMO) systems is deduced with the aid of a simplified approach that relies on a fundamental power normalization. Given their high practical usability, we are particularly interested in dual configurations where both ends deploy two antenna elements. Contrary to the majority of related studies, where only the common case of Rayleigh fading is considered, our analysis is extended to account for Ricean fading where a deterministic Line-of-Sight (LoS) component exists in the communication link and both ends are affected by spatial correlation. In the following, it is shown that the proposed bound is applicable for any arbitrary Signal-to-Noise Ratio (SNR) and rank of the mean channel matrix. In fact, we consider both conventional and optimized MIMO configurations with equal LoS eigenvalues. Moreover, the tightness of the bound is explored where it is demonstrated that as the SNR tends to zero the bound becomes asymptotically tight; at high SNRs, the offset between empirical capacity and the bound is analytically computed which implies that an explicit asymptotic capacity expression can ultimately be obtained.
\end{abstract}

Index Terms-MIMO systems, ergodic capacity, Ricean fading, spatial fading correlation.

\section{INTRODUCTION}

$\mathbf{O}$ VER the last years, a considerable amount of research interest has been devoted to Multiple-Input MultipleOutput (MIMO) systems in response to the increasing demand for higher data rates. The pioneering works of Foschini [1] and Telatar [2] demonstrated the dramatic performance enhancement when multiple antenna elements are employed at both ends of a radio link. In order to comprehend the advantages of this promising technology, one of the most interesting topics that needs to be addressed is the derivation of efficient analytical capacity bounds. By doing so, it is anticipated that the design of practical and simulated MIMO systems would be greatly enriched. Please note that throughout the paper our main interest will lie in dual MIMO configurations which are likely to be used in many future practical systems

Paper approved by N. Jindal, the Editor for MIMO Techniques of the IEEE Communications Society. Manuscript received August 1, 2008; revised April 4, 2009.

M. Matthaiou is with the Institute for Circuit Theory and Signal Processing, Technische Universität München (TUM), Arcistrasse 21, 80333, Munich, Germany (e-mail: matthaiou@nws.ei.tum.de).

Y. Kopsinis and D. I. Laurenson are with the Institute for Digital Communications (IDCOM), Joint Research Institute for Signal and Image Processing, The University of Edinburgh, EH9 3JL, Edinburgh, U.K. (e-mail: \{Y.Kopsinis, Dave.Laurenson\} @ed.ac.uk).

A. M. Sayeed is with the Department of Electrical and Computer Engineering, University of Wisconsin-Madison, Madison, WI 53706 USA (e-mail: akbar@engr.wisc.edu).

Digital Object Identifier 10.1109/TCOMM.2009.12.080393 (e.g. hand-held devices), thanks to their small size and low implementation cost.

The majority of related studies documented in literature consider the tractable case of Rayleigh fading where no direct Line-of-Sight (LoS) exists in the radio channel and the multipath richness is sufficiently high. In this case, a plethora of results is available for various scenarios (see [1][4] and references therein). In most real-life channels though, the assumption of Rayleigh fading is often violated due to either a specular wavefront or a strong direct component; then, the entries of the channel matrix can be more effectively modeled by the Ricean distribution. Surprisingly, significantly fewer publications focusing on MIMO Ricean channels have been reported. This trend can be attributed to the difficulty in manipulating hypergeometric functions with two matrix arguments of non-central Wishart matrices compared to the one matrix argument of central (zero-mean) Wishart matrices tied to Rayleigh fading.

The first analytical bounds on MIMO Ricean capacity can be found in [5]-[7] where the assumption of uncorrelated fading at both ends was adopted. In [8], [9], these results were extended to account for spatial correlation at a single end while the general case of double-sided spatial correlation was addressed in [10]. The common characteristic of the above cited papers though ([5]-[10]), is that they are limited to the case of rank-1 LoS matrices. The derivation of capacity bounds in the general case of arbitrary-rank mean matrices has been separately addressed in [11]-[14]. In [11] and [12], very tight lower and upper capacity bounds for semi-correlated MIMO Ricean channels were respectively proposed. The work in [13] represents so far the more general approach in the area since it derives several lower and upper bounds assuming all different types of spatial correlation. However, the general upper bound is given as an infinite summation of Hayakawa polynomials, which the authors acknowledge as quite involved and computationally inefficient. On the other hand, the upper bound deduced in [14], which relies on the theory of quadratic forms, is far more tractable and hence will serve as our reference bound hereafter. Whilst the authors in [14] focus on the general properties of quadratic forms with respect to MIMO capacity bounds, our primary goal is to provide a simplified bound derivation and thereafter investigate the implications of the model parameters (Ricean $K$-factor and spatial correlation) on the bound performance.

More specifically, the contributions of the present paper are threefold. Firstly, an alternative derivation of the upper bound in [14] is proposed for the dual MIMO case under 
consideration. Our analysis relies on a well-established power normalization scheme which simplifies significantly all the mathematical formulations. Secondly, using the same normalization as a starting point, the asymptotic tightness of the upper bound is explored in the low and high Signal-to-Noise Ratio (SNR) regimes. In fact, it is explicitly shown that as the SNR tends to zero the bound becomes asymptotically tight, whereas, at infinitely high SNRs the offset from the true capacity is analytically determined as well; hence, exact capacity expressions can be derived in the corresponding asymptotic cases. Finally, in order to formulate a broad framework, two different classes of LoS MIMO systems are assessed, namely a conventional low-rank (LR) and an optimized high-rank (HR) configuration which yields two equal LoS eigenvalues and is of high practical importance [15]-[17].

The paper is organized as follows: In Section II, some essential definitions related to non-central Wishart matrices and quadratic forms are given. In Section III, the underlying MIMO Ricean channel model is presented along with the statistics of the channel matrix. Section IV presents upper capacity bounds for different categories of MIMO systems. In Section V, we focus on the tightness of the proposed bound while the numerical results are given in Section VI. Finally, Section VII summarizes the key findings.

Notation: We use upper and lower case boldfaces to denote matrices and vectors. The symbol $\sim \mathcal{C N}_{m, n}(\mathbf{M}, \boldsymbol{\Sigma})$ stands for a $m \times n$ complex normally distributed matrix with mean $\mathbf{M}$ and covariance $\boldsymbol{\Sigma}$. The entries of a $m \times n$ matrix $\mathbf{A}$ are given by $\{\mathbf{A}\}_{i, j}$ where $i=1, \ldots, m$ and $j=1, \ldots, n$. The symbols $(\cdot)^{T},(\cdot)^{H}$ and $(\cdot)^{-1}$ correspond to transposition, Hermitian transposition and matrix inversion respectively whereas $\otimes$ is the Kronecker product; we use $\operatorname{det}(\cdot)$ and $|\cdot|$ to interchangeably denote the determinant while $\|\cdot\|_{F}$ returns the Frobenius norm. Finally, $\operatorname{etr}(\cdot)$ is a shorthand notation for $\exp (\operatorname{tr}(\cdot))$.

\section{COMPlex Non-Central Wishart Matrices And QUADRATIC FORMS}

Definition 1: Let the $m \times n$ complex matrix $\mathbf{X}$, with $m \leq n$, be distributed according to $\mathbf{X} \sim \mathcal{C N}_{m, n}\left(\mathbf{M}, \boldsymbol{\Sigma} \otimes \mathbf{I}_{n}\right)$, where $\boldsymbol{\Sigma} \in \mathbb{C}^{m \times m}$ is a positive definite Hermitian matrix. Then, the matrix $\mathbf{S}=\mathbf{X X}^{H}$ follows the complex non-central Wishart distribution with $n$ degrees of freedom and noncentrality matrix $\Omega=\boldsymbol{\Sigma}^{-1} \mathbf{M} \mathbf{M}^{H}$, commonly denoted as $\mathbf{S} \sim \mathcal{C W}_{m}(n, \boldsymbol{\Sigma}, \boldsymbol{\Omega})$.

Definition 2: Let the $m \times n$ complex matrix $\mathbf{X}$, with $m \leq n$, be distributed according to $\mathbf{X} \sim \mathcal{C N}_{m, n}(\mathbf{M}, \boldsymbol{\Sigma} \otimes \boldsymbol{\Psi})$, where $\boldsymbol{\Sigma} \in \mathbb{C}^{m \times m}$ and $\boldsymbol{\Psi} \in \mathbb{C}^{n \times n}$ are positive definite Hermitian matrices. Then, the matrix $\mathbf{Q}=\mathbf{X} \mathbf{\Lambda} \mathbf{X}^{H}$, with $\boldsymbol{\Lambda} \in \mathbb{C}^{n \times n}$, follows a non-central matrix-variate quadratic form commonly denoted as $\mathbf{Q} \sim \mathcal{C} \mathcal{Q}_{m, n}(\boldsymbol{\Lambda}, \boldsymbol{\Sigma}, \boldsymbol{\Psi}, \mathbf{M})$.

Please note that non-central quadratic forms degenerate into non-central Wishart matrices when $\boldsymbol{\Psi}=\mathbf{I}_{n}$ and when either $\boldsymbol{\Lambda}=\mathbf{I}_{n}$ or $\boldsymbol{\Lambda}$ is idempotent with rank $L \geq m$ [13]. The following theorem returns the $v$-th moment of the determinant of $2 \times 2$ complex quadratic forms.

Theorem 1: Let $\mathbf{Q} \sim \mathcal{C Q}_{2,2}\left(\mathbf{I}_{2}, \boldsymbol{\Sigma}, \mathbf{\Psi}, \mathbf{M}\right)$. Then, the $v$-th moment of its determinant $|\mathbf{Q}|$ is given by

$$
E\left[|\mathbf{Q}|^{v}\right]=|\boldsymbol{\Sigma} \boldsymbol{\Psi}|^{v}\left[\frac{\tilde{\Gamma}_{2}(v+2)}{\tilde{\Gamma}_{2}(2)}\right]{ }_{1} \widetilde{F}_{1}(-v ; 2 ;-\mathbf{\Theta})
$$

where $\tilde{\Gamma}_{m}(n)=\pi^{m(m-1) / 2} \prod_{j=1}^{m} \Gamma(n-j+1)$ is the complex multivariate gamma function, with $\Gamma(\cdot)$ being the standard gamma function, while ${ }_{1} \widetilde{F}_{1}(a, b, \mathbf{A})$ is the hypergeometric function of one matrix argument; the involved matrices are accordingly $\boldsymbol{\Theta}=\boldsymbol{\Psi}^{-1} \overline{\mathbf{M}} \overline{\mathbf{M}}^{H}$ and $\overline{\mathbf{M}}=\boldsymbol{\Sigma}^{-1 / 2} \mathbf{M}$.

Proof: A detailed proof is given in Appendix A.

It should be noted that the theorem is applicable only to $2 \times 2$ quadratic forms since for matrix sizes of $m \times n$, a finite summation over a collection of $\left(\begin{array}{l}n \\ m\end{array}\right)$ subsets needs to take place [14]. A simplified formula can now be obtained for the first-order moment of the determinant.

Corollary 1: For $v=1,(1)$ reduces to

$$
E[|\mathbf{Q}|]=2|\boldsymbol{\Sigma} \boldsymbol{\Psi}|\left(1+\frac{1}{2} \operatorname{tr}(\boldsymbol{\Theta})+\frac{1}{2} \operatorname{det}(\boldsymbol{\Theta})\right) .
$$

Proof: The authors in [13] showed that for any square matrix $\mathbf{B} \in \mathbb{C}^{m \times m}$, its hypergeometric function ${ }_{1} \widetilde{F}_{1}(c ; d ; \mathbf{B})$ can be expressed according to

$$
{ }_{1} \widetilde{F}_{1}(c ; d ; \mathbf{B})=\frac{\operatorname{det}\left({ }_{1} \widetilde{F}_{1}\left(c-m+j ; d-m+j ; b_{i}\right) b_{i}^{j-1}\right)}{\prod_{i<j}^{m}\left(b_{j}-b_{i}\right)}
$$

where $b_{1}, b_{2}, \ldots, b_{m}$ is the set of non-zero eigenvalues of $\mathbf{B}$. After taking into account the following properties of the scalar hypergeometric functions

$$
\begin{aligned}
{ }_{1} \widetilde{F}_{1}(-2,1 ; z) & =1-2 z+\frac{1}{2} z^{2} \\
{ }_{1} \widetilde{F}_{1}(-1,2 ; z) & =1-\frac{1}{2} z^{2}
\end{aligned}
$$

it is trivial to show that for the dual case under consideration

$$
{ }_{1} \widetilde{F}_{1}(-1 ; 2 ;-\boldsymbol{\Theta})=\frac{1}{2}\left(2+\theta_{1}+\theta_{2}+\theta_{1} \theta_{2}\right)
$$

where $\theta_{1}, \theta_{2}$ are the eigenvalues of $\boldsymbol{\Theta}$. The proof concludes after recalling that the sum and product of the eigenvalues return the trace and the determinant of a matrix.

\section{MIMO CHANNEL MODEL}

As was previously highlighted, we are particularly interested in dual MIMO configurations where both the Tx and Rx are affected by spatial correlation. Under Ricean fading conditions, the channel transfer function matrix $\mathbf{H}$ consists of a spatially deterministic specular component $\mathbf{H}_{\mathrm{L}}$ and a randomly distributed component $\mathbf{H}_{\mathrm{W}}$ which accounts for the scattered signals. Then, the underlying channel model becomes

$$
\mathbf{H}=\sqrt{\frac{K}{K+1}} \mathbf{H}_{\mathrm{L}}+\sqrt{\frac{1}{K+1}} \mathbf{R}_{r}^{1 / 2} \mathbf{H}_{\mathrm{W}} \mathbf{R}_{t}^{1 / 2}
$$

where $K$ is the Ricean $K$-factor expressing the ratio of powers of the free-space signal and the scattered waves. The receive and transmit correlation matrices are respectively defined as

$$
\mathbf{R}_{r} \triangleq E_{\mathbf{H}}\left\{\mathbf{H} \mathbf{H}^{H}\right\} \text { and } \mathbf{R}_{t} \triangleq E_{\mathbf{H}}\left\{\left(\mathbf{H}^{H} \mathbf{H}\right)^{T}\right\}
$$




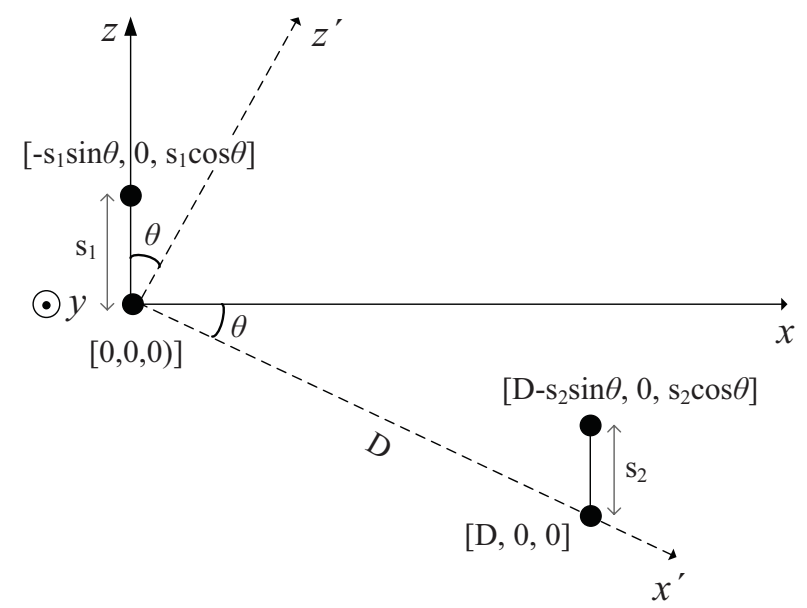

Fig. 1. A general architecture of a $2 \times 2$ MIMO system with ULAs at both ends (side view). The coordinates of all elements with regard to the new coordinate system $x^{\prime} y z^{\prime}$ are also included. The axis rotation by an angle $\theta$ arranges the array origins to lie on the same axis.

The entries of $\mathbf{H}_{\mathrm{W}}$ are commonly modeled as i.i.d. zeromean, unit variance complex Gaussian random variables and, consequently, the channel matrix is distributed according to

$$
\mathbf{H} \sim \mathcal{C N}_{2,2}\left(\sqrt{K /(K+1)} \mathbf{H}_{\mathrm{L}},(K+1)^{-1} \mathbf{R}_{r} \otimes \mathbf{R}_{t}\right) .
$$

Throughout the paper, our purpose is to compare the MIMO gains of different configurations and therefore the capacities should be analyzed independently of the average SNR. This is achieved by normalizing the channel matrices so that following condition is fulfilled

$$
E\left[\|\mathbf{H}\|_{F}^{2}\right]=E\left[\operatorname{tr}\left(\mathbf{H} \mathbf{H}^{H}\right)\right]=4 .
$$

We recall that the key normalization in (10) has been widely adopted into the capacity characterization of MIMO systems [1], [2], [12], [16], [17]. From a physical viewpoint, the path-loss effects are removed and the system is assumed to have perfect power control.

In Fig. 1, a side view of the considered MIMO system is depicted where both ends are equipped with two-element Uniform Linear Arrays (ULAs) and the distance between the first element of each array is $D$. The inter-element spacings are $s_{1}(\mathrm{Tx})$ and $s_{2}(\mathrm{Rx})$.

In the following, we will explore two different LoS configurations, namely a LR and an optimized HR configuration. The former represents any conventional architecture with interelement spacings of the order of wavelength $\lambda$, and arrays located in each other's far-field; this means that the LoS signals are basically plane wavefronts whose rays' phases are inherently correlated to a great extent. These architectures are normally rank-deficient and offer a minimal spatial multiplexing gain since the transmitted LoS signals impinging on the $\mathrm{Rx}$ carry almost identical spatial characteristics and therefore their differentiation becomes laborious. The latter configuration belongs to a family of specifically designed full-rank LoS configurations which aim to assign a phase difference of $\pi / 2$ between the LoS wavefronts [15]-[17]. This is achieved by appropriately positioning the antenna elements at both ends of the link, so that the LoS signals propagate as spherical wavefronts in the near-field. Then, the channel matrix becomes full-rank and delivers two equal LoS eigenvalues. For the assumed model, the optimum inter-element spacing is [17]

$$
s_{1}=s_{2}=s_{o p t} \approx \sqrt{\frac{\lambda D}{2 \cos ^{2} \theta}} .
$$

We emphasize that for optimized configurations the effects of spatial correlation are rather weak due to the increased antenna spacing. However, in the following analysis the presence of correlation is considered, for the fairness of comparison with conventional configurations.

\section{ERGOdic CAPACITY UPPER BoUNDS}

In this section, an alternative simplified derivation for the upper bound of dual MIMO configurations (as given in [14]) is presented based on the power normalization of (10). Assuming that the Rx has perfect CSI while the Tx knows neither the statistics nor the instantaneous CSI, a sensible choice for the Tx is to split the total amount of power equally amongst all data streams. Then, the ergodic MIMO capacity for an SNR per receiver branch, $\rho$, is given by the following well-known formula [1], [2]

$$
\bar{C}=E_{\mathbf{H}}\left[\log _{2}\left(\operatorname{det}\left(\mathbf{I}_{2}+\frac{\rho}{2} \mathbf{H H}^{H}\right)\right)\right] \quad(\text { bits } / \mathrm{s} / \mathrm{Hz}) .
$$

\section{A. Double-Sided Correlated Ricean and Rayleigh Fading}

In the general case of double-sided correlated Ricean fading, where the channel matrix is distributed according to (9), the instantaneous MIMO correlation matrix $\widetilde{\mathbf{W}}=\mathbf{H H}^{H}$ exhibits the following non-central quadratic distribution

$$
\widetilde{\mathbf{W}} \sim \mathcal{C} \mathcal{Q}_{2,2}\left(\mathbf{I}_{2}, \mathbf{R}_{r} /(K+1), \mathbf{R}_{t}, \sqrt{K /(K+1)} \mathbf{H}_{\mathrm{L}}\right) .
$$

Theorem 2: The ergodic capacity of a $2 \times 2$ doublesided correlated MIMO Ricean channel with mean matrix $\sqrt{K /(K+1)} \mathbf{H}_{\mathrm{L}}$, receive correlation matrix $(K+1)^{-1} \mathbf{R}_{r}$, and transmit correlation matrix $\mathbf{R}_{t}$, is analytically upper bounded by

$$
\bar{C} \leq \log _{2}\left(1+2 \rho+\frac{\gamma \rho^{2}}{2}\right)
$$

where the parameter $\gamma$ and the matrix $\Theta$ are respectively

$$
\begin{array}{r}
\gamma=(K+1)^{-2}\left|\mathbf{R}_{r} \| \mathbf{R}_{t}\right|\left(1+\frac{1}{2} \operatorname{tr}(\boldsymbol{\Theta})+\frac{1}{2} \operatorname{det}(\boldsymbol{\Theta})\right) \\
\boldsymbol{\Theta}=K \mathbf{R}_{t}^{-1} \mathbf{R}_{r}^{-1 / 2} \mathbf{H}_{\mathrm{L}} \mathbf{H}_{\mathrm{L}}^{H}\left(\mathbf{R}_{r}^{-1 / 2}\right)^{H} .
\end{array}
$$

Proof: An alternative way to express the ergodic MIMO capacity is through the real positive eigenvalues $\widetilde{w}_{1}, \widetilde{w}_{2}$ of $\widetilde{\mathbf{W}}$. Then, (12) can be rewritten as

$$
\bar{C}=E\left[\log _{2}\left(1+\frac{\rho}{2} \widetilde{w}_{1}\right)\left(1+\frac{\rho}{2} \widetilde{w}_{2}\right)\right] .
$$

Expanding (17), we can easily get

$$
\bar{C}=E\left[\log _{2}\left(1+\frac{\rho}{2}\left(\widetilde{w}_{1}+\widetilde{w}_{2}\right)+\frac{\rho^{2}}{4} \operatorname{det}(\widetilde{\mathbf{W}})\right)\right] .
$$


Taking into account that $\log (\cdot)$ is a concave function and making use of the Jensen's inequality we obtain

$$
\bar{C} \leq \log _{2}\left(1+2 \rho+\frac{\rho^{2}}{4} E[\operatorname{det}(\widetilde{\mathbf{W}})]\right) .
$$

The last inequality follows from the power normalization in (10), i.e. $E[\operatorname{tr}(\widetilde{\mathbf{W}})]=E\left[\widetilde{w}_{1}+\widetilde{w}_{2}\right]=4$. The upper bound in (14) follows immediately after introducing Corollary 1 and simplifying.

It is worth mentioning that the same upper capacity bound for $2 \times 2$ MIMO systems was originally given in [14]. More specifically, by setting $n=2$ in [14, Eq. (36)] and evaluating the first three basic elementary functions and minor determinant representations involved, the same result can be obtained after some algebraic manipulations. In the present case though, a novel derivation is introduced, through the power normalization of (10), which simplifies extensively the overall analysis. Please note that the proposed derivation is tied to dual configurations since for larger MIMO setups we end up with a series of eigenvalue cross-products that are created after expanding (12). By inspection of (14), it can be inferred that the proposed upper bound is rather simple as it just requires the computation of the elementary functions of three different deterministic matrices $\left(\mathbf{R}_{r}, \mathbf{R}_{t}\right.$ and $\left.\boldsymbol{\Theta}\right)$ only once.

In the case of double-sided correlated Rayleigh fading ( $K=0$ ), the channel matrix is distributed according to $\mathbf{H} \sim \mathcal{C N}_{2,2}\left(\mathbf{0}_{2}, \mathbf{R}_{r} \otimes \mathbf{R}_{t}\right)$ and the upper bound in (14) reduces to

$$
\bar{C} \leq \log _{2}\left(1+2 \rho+\frac{\rho^{2}}{2}\left|\mathbf{R}_{r} \mathbf{R}_{t}\right|\right)
$$

which is in perfect line with the bound derived in [4, Eq. (25)].

\section{B. Uncorrelated Ricean and Rayleigh Fading}

Due to space constraints, the case of single-side correlation is omitted herein since the formulations are based on exactly the same concept as before. We now consider the special case of both ends exhibiting uncorrelated i.i.d. Ricean fading. Under these circumstances, the channel matrix is distributed as

$$
\mathbf{H} \sim \mathcal{C N}_{2,2}\left(\sqrt{\frac{K}{K+1}} \mathbf{H}_{\mathrm{L}}, \frac{1}{K+1} \mathbf{I}_{2} \otimes \mathbf{I}_{2}\right) .
$$

Corollary 2: The ergodic capacity of a $2 \times 2$ uncorrelated MIMO Ricean channel with mean matrix $\sqrt{K /(K+1)} \mathbf{H}_{\mathrm{L}}$ and receive correlation matrix $\mathbf{R}_{r}=(K+1)^{-1} \mathbf{I}_{2}$ is analytically upper bounded by

$$
\bar{C} \leq \log _{2}\left(1+2 \rho+\frac{\beta \rho^{2}}{2}\right)
$$

where $\beta=\left(1+2 K+0.5 K^{2} \operatorname{det}(\mathbf{T})\right) /(K+1)^{2}$ and $\mathbf{T}=$ $\mathbf{H}_{\mathrm{L}} \mathbf{H}_{\mathrm{L}}^{H}$.

Proof: This corollary is a direct consequence of (14) after taking into account that $\Theta \equiv \Omega \equiv K \mathbf{T}$ for the case of uncorrelated fading at both ends. Furthermore, it holds that $\operatorname{tr}(K \mathbf{T})=K \operatorname{tr}(\mathbf{T})$ and $\operatorname{det}(K \mathbf{T})=K^{2} \operatorname{det}(\mathbf{T})$ and given that the entries of the deterministic LoS component matrix are normally modeled as unit-amplitude complex exponentials [15]-[17], it is trivial to show that $\operatorname{tr}(\mathbf{T})=4$.
The case of i.i.d. Rayleigh fading is obtained directly from (7), by setting $K=0$ and $\mathbf{R}_{r}=\mathbf{R}_{t}=\mathbf{I}_{2}$. The upper bound in (22) then reduces to $(\beta=1)$

$$
\bar{C} \leq \log _{2}\left(1+2 \rho+\frac{\rho^{2}}{2}\right)
$$

which is identical with the results presented in $[3$, Theorem 2], [4, Eq. (22)] and [12, Eq. (5)].

\section{Tightness OF THE UPPER BOUND}

In this section, the asymptotic tightness of the proposed upper bound is investigated in low and high-SNR regimes. To this end, we define the absolute error, $\epsilon$, inserted by an upper bound, $U$, as $\epsilon=U-\bar{C}$.

Corollary 3: The upper bound in (14) becomes asymptotically tight as the SNR tends to zero.

Proof: We begin with further upper bounding the ergodic capacity (14) recalling that $\ln (1+x) \leq x$

$$
\bar{C} \leq \frac{1}{\ln 2}\left(2 \rho+\frac{\gamma \rho^{2}}{2}\right) .
$$

Following [18, Eq. (23)], we can lower bound the ergodic capacity as

$$
\begin{aligned}
\bar{C} & \geq E\left[\log _{2}\left(1+\frac{\rho}{2}\|\mathbf{H}\|_{F}^{2}\right)\right] \\
& \geq \frac{\rho}{2 \ln 2} E\left[\|\mathbf{H}\|_{F}^{2}\right]-\frac{1}{2 \ln 2}\left(\frac{\rho}{2}\right)^{2} E\left[\|\mathbf{H}\|_{F}^{4}\right] .
\end{aligned}
$$

The second line follows from the property $\ln (1+x) \geq x-\frac{1}{2} x^{2}$. We can now subtract (25) from (24) and then the absolute error $\epsilon$ of the proposed upper bound becomes

$$
\epsilon=\frac{\rho^{2}}{2 \ln 2}\left(\gamma+\frac{1}{4} E\left[\|\mathbf{H}\|_{F}^{4}\right]\right)
$$

which asymptotically tends to zero as $\rho \rightarrow 0$.

Corollary 4: As the SNR $\rho \rightarrow \infty$, the absolute error inserted by the upper bound in (14) tends to

$$
\epsilon=\log _{2}(2 \gamma)-E\left[\log _{2}(\operatorname{det}(\widetilde{\mathbf{W}}))\right] .
$$

Proof: As $\rho \rightarrow \infty$, the upper bound $U$ in (14), becomes

$$
U \approx \log _{2}(2 \gamma)+2 \log _{2}\left(\frac{\rho}{2}\right) .
$$

In (18), the quadratic term becomes significantly larger at high SNR and thus the ergodic capacity may be approximated as

$$
\begin{aligned}
\bar{C} & \approx E\left[\log _{2}\left(\frac{\rho^{2}}{4} \operatorname{det}(\widetilde{\mathbf{W}})\right)\right] \\
& =2 \log _{2}\left(\frac{\rho}{2}\right)+E\left[\log _{2}(\operatorname{det}(\widetilde{\mathbf{W}}))\right] .
\end{aligned}
$$

Subtracting (29) from (28), yields (27).

From the above equation, it is apparent that the bound's error is given in a non-analytical form; in this light, the crucial issue is to determine the expectation of the logdet function of a complex non-central quadratic matrix, which involves a nonlinear log function. 
Theorem 3: Let us assume that the considered matrix $\widetilde{\mathbf{W}} \sim \mathcal{C} \mathcal{Q}_{2,2}\left(\mathbf{I}_{2}, \mathbf{R}_{r} /(K+1), \mathbf{R}_{t}, \sqrt{K /(K+1)} \mathbf{H}_{\mathrm{L}}\right)$. Then the first-order moment of the logarithm of its determinant is

$$
\begin{aligned}
E\left[\log _{2}(\operatorname{det}(\widetilde{\mathbf{W}}))\right] & =\frac{1}{\ln 2}[\psi(1)+\psi(2)-2 \ln (K+1) \\
+\ln \left|\mathbf{R}_{r} \mathbf{R}_{t}\right| & \left.-\frac{1}{\theta_{1}-\theta_{2}}\left(\Lambda_{1}(\boldsymbol{\Theta})+\Lambda_{2}(\boldsymbol{\Theta})\right)\right]
\end{aligned}
$$

where $\theta_{1}, \theta_{2}$ are the eigenvalues of $\boldsymbol{\Theta}$, given in (16), while the digamma functions $\psi(x)$ are defined as

$$
\psi(x) \triangleq \frac{d}{d x} \ln \Gamma(x)=\frac{\Gamma^{\prime}(x)}{\Gamma(x)} .
$$

The polynomial terms $\Lambda_{1}(\boldsymbol{\Theta})$ and $\Lambda_{2}(\boldsymbol{\Theta})$ are essentially polynomial functions of the eigenvalues $\theta_{1}$ and $\theta_{2}$, or

$$
\begin{aligned}
& \Lambda_{1}(\boldsymbol{\Theta})=\theta_{2} h_{1}\left(\theta_{1}\right)-h_{2}\left(\theta_{1}\right) \\
& \Lambda_{2}(\boldsymbol{\Theta})=h_{2}\left(\theta_{2}\right)-\theta_{1} h_{1}\left(\theta_{2}\right)
\end{aligned}
$$

where

$$
h_{1}(x)=\sum_{k=1}^{\infty} \frac{\mathcal{P}(k, x)}{k}, \quad h_{2}(x)=x \sum_{k=1}^{\infty} \frac{\mathcal{P}(k, x)}{k+1}
$$

with $\mathcal{P}(a, x)$ being the regularized gamma function [19, Eq. (6.5.1)].

Proof: A detailed proof is given in Appendix B.

Please note that the derivation of the formula (30) relies, without loss of generality, on the assumption of two non-zero eigenvalues of the matrix $\Theta$. For the case of rank-1 matrices, a similar analysis can be followed and the interested readers are referred to [13] for a thorough discussion. Evidently, after replacing (30) into (27), we can obtain an analytical formula for the bound's offset at high SNRs, for the general case of double-sided correlated Ricean fading. More importantly, this result can be further used to deduce exact capacity expressions in the high-SNR regime.

When the channel exhibits i.i.d. Ricean fading and both ends are employed with optimally designed arrays, the LoS component yields two equal eigenvalues and the following corollary is introduced.

Corollary 5: As the SNR $\rho \rightarrow \infty$, the absolute error inserted by the upper bound for the case of i.i.d. Ricean fading and optimized LoS configurations tends to

$$
\begin{aligned}
\epsilon=\log _{2}(2 \beta) & -\frac{1}{\ln 2}[\psi(1)+\psi(2)-2 \ln (K+1) \\
& \left.+\sum_{k=1}^{\infty} \frac{(2 k+1) \gamma(k, \omega)-\omega e^{-\omega} \omega^{k-1}}{(k+1) !}\right]
\end{aligned}
$$

where $\omega=\omega_{1}=\omega_{2}$ represents any of the two equal eigenvalues of $\boldsymbol{\Omega} \equiv K \mathbf{T}$ and $\gamma(a, x)=\int_{0}^{x} t^{a-1} e^{-t} d t$ is the lower incomplete gamma function.

Proof: The proof starts by noting that for i.i.d. Ricean fading $\Theta$ should be replaced by $\Omega \equiv K \mathbf{T}$ in all manipulations. Further, in the specific case of optimized configurations, the equality of eigenvalues leads to a division by zero in (30). In order to circumvent this singularity, we employ de l'Hôpital's

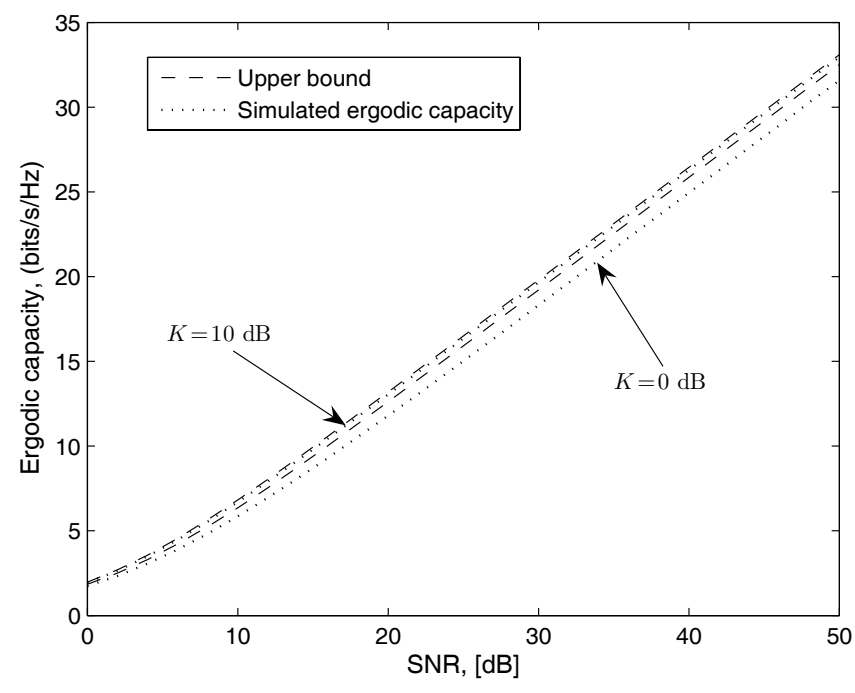

Fig. 2. Upper bound and simulated ergodic capacity as a function of the SNR for an optimized $\operatorname{LoS}$ configuration $\left(\delta_{R}=0.2\right.$ and $\left.\delta_{T}=0.5\right)$.

rule to get a solution at the limit $\omega_{1} \rightarrow \omega_{2}$. In particular, the last term in (30) can be rewritten after some algebra as

$$
\lim _{\varepsilon \rightarrow 0}\left[\frac{d}{d \varepsilon}\left(\omega h_{1}(\omega+\varepsilon)-h_{2}(\omega+\varepsilon)\right)\right]-h_{1}(\omega) .
$$

Taking into account that

$$
\frac{d}{d x} \mathcal{P}(a, x)=\frac{e^{-x} x^{a-1}}{\Gamma(a)}
$$

and after some algebraic manipulations we end up with

$$
\begin{aligned}
& \lim _{\varepsilon \rightarrow 0} \frac{d}{d \varepsilon} h_{1}(\omega+\varepsilon)=\sum_{k=1}^{\infty} \frac{e^{-\omega} \omega^{k-1}}{k \Gamma(k)} \\
& \lim _{\varepsilon \rightarrow 0} \frac{d}{d \varepsilon} h_{2}(\omega+\varepsilon)=\sum_{k=1}^{\infty} \frac{e^{-\omega} \omega^{k-1}}{(k+1) \Gamma(k)}+\frac{h_{2}(\omega)}{\omega} .
\end{aligned}
$$

Substituting (38)-(39) into (36), factorizing and simplifying yields (35).

\section{Numerical Results}

In this section, the theoretical analysis presented in Sections IV and V is validated through a set of Monte-Carlo simulations. Assuming a carrier frequency of $5.9 \mathrm{GHz}, D=5.3852$ $\mathrm{m}$ and $\theta=21.80^{\circ}$ the optimum inter-element spacings via (11) are $s_{1}=s_{2}=39.85 \mathrm{~cm}(7.84 \lambda)$ whereas for the conventional configuration the spacings are taken $s_{1}=s_{2}=2.54 \mathrm{~cm}$ $(0.5 \lambda)$. Then, the associated sets of the LoS eigenvalues of $\mathbf{T}=\mathbf{H}_{\mathrm{L}} \mathbf{H}_{\mathrm{L}}^{H}$ are $(2,2)$ and $\left(3.999,4.08 \times 10^{-5}\right)$, respectively. Throughout the simulations, we adopt the widely used exponential correlation model thanks to its inherent simplicity. Then, the entries of $\mathbf{R}_{r}$ and $\mathbf{R}_{t}$ in (7) can be modeled as $\left\{\mathbf{R}_{r}\right\}_{i, j}=\left(\delta_{R}\right)^{|i-j|}$ and $\left\{\mathbf{R}_{t}\right\}_{i, j}=\left(\delta_{T}\right)^{|i-j|}$, where $\delta_{R}, \delta_{T} \in[0,1)$.

After generating 50,000 Monte-Carlo realizations of the channel matrix according to (7) and setting $\delta_{R}=0.2$ and $\delta_{T}=0.5$, the proposed bound in (14) is firstly evaluated against the SNR for different values of the $K$-factor. 


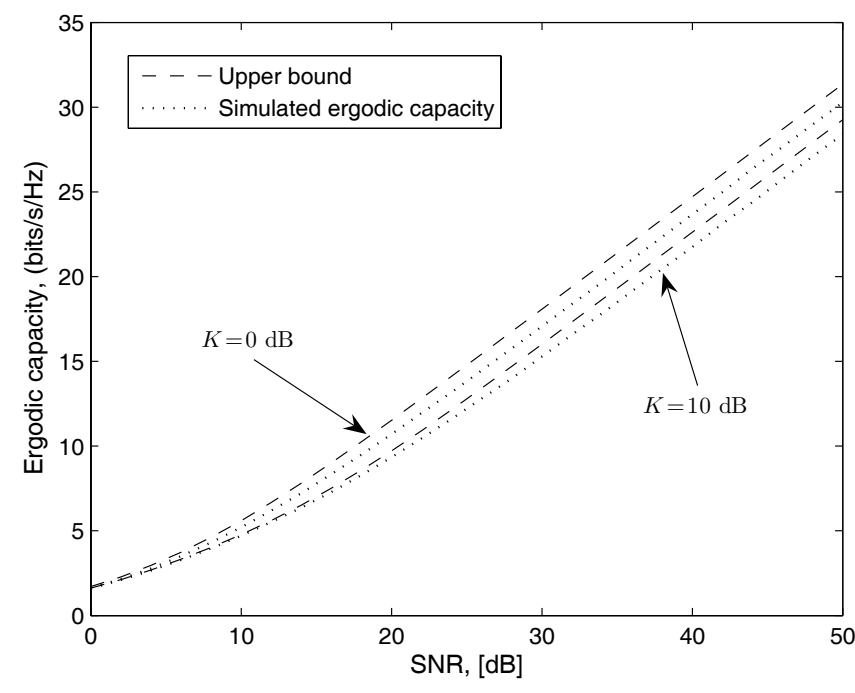

Fig. 3. Upper bound and simulated ergodic capacity as a function of the SNR for a conventional LoS configuration $\left(\delta_{R}=0.2\right.$ and $\left.\delta_{T}=0.5\right)$.

From Fig. 2 and 3, it can been seen that the bound is remarkably tight for the optimized configuration and, likewise, performs satisfactorily for conventional configurations. As anticipated, in the low-SNR regime both bounds converge asymptotically to the empirical values of ergodic capacity. Generally speaking, the bound becomes tighter as $K$ increases and SNR decreases which is in agreement with the conclusions of [9], [10], [12]. More importantly, ergodic capacity benefits from the presence of strong non-fading components when both ends are equipped with specifically designed arrays. This is a result of the two orthogonal LoS MIMO subchannels that are established and contradicts the common belief that high $K$-factors limit the advantages of MIMO technology [6], [7], [9].

In Fig. 4, the effects of the $K$-factor on the performance of the proposed bound are investigated, where it is again apparent that both bounds become tighter with an increasing $K$-factor. For Rayleigh-fading conditions though, or $K \leq 0 \mathrm{~dB}$, the achieved tightness is degraded and, under these circumstances, it is sensible to use more efficient bounds which are inherently tied to Rayleigh channels (see for instance [3], [4]). Once more, the superiority of the optimized configurations is illustrated as $K$ gets larger whereas in the limit, $K \rightarrow \infty \mathrm{dB}$, conventional configurations degenerate into a single-path link. On the other hand, for $K \leq 0 \mathrm{~dB}$ the advantages of optimized configurations diminish and in the limit, $K \rightarrow-\infty \mathrm{dB}$, the LoS component vanishes and we end up with a pure i.i.d. Rayleigh channel.

In Fig. 5, the relationship between spatial correlation and MIMO capacity is addressed. The effects of the former become less significant (smaller dynamic range) as the $K$ factor gets higher for both configurations, i.e. high $K$-factors provide robustness against spatial correlation. As expected, the optimized setup with large inter-element spacings remains unaffected by the level of correlation; hence, it offers almost the same ergodic capacity regardless of the values of $\delta_{R}$ and $\delta_{T}$. The conventional configuration, however, suffers from spatial correlation with the ergodic capacity decreasing as

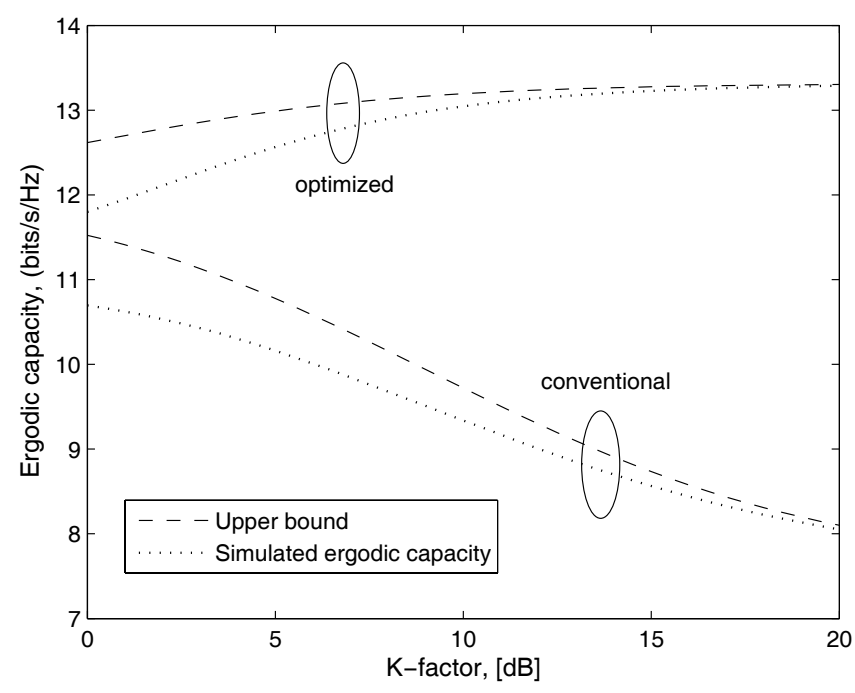

Fig. 4. Upper bound and simulate ergodic capacity as a function of the $K$-factor $\left(\delta_{R}=0.2, \delta_{T}=0.5\right.$ and $\left.\rho=20 \mathrm{~dB}\right)$.

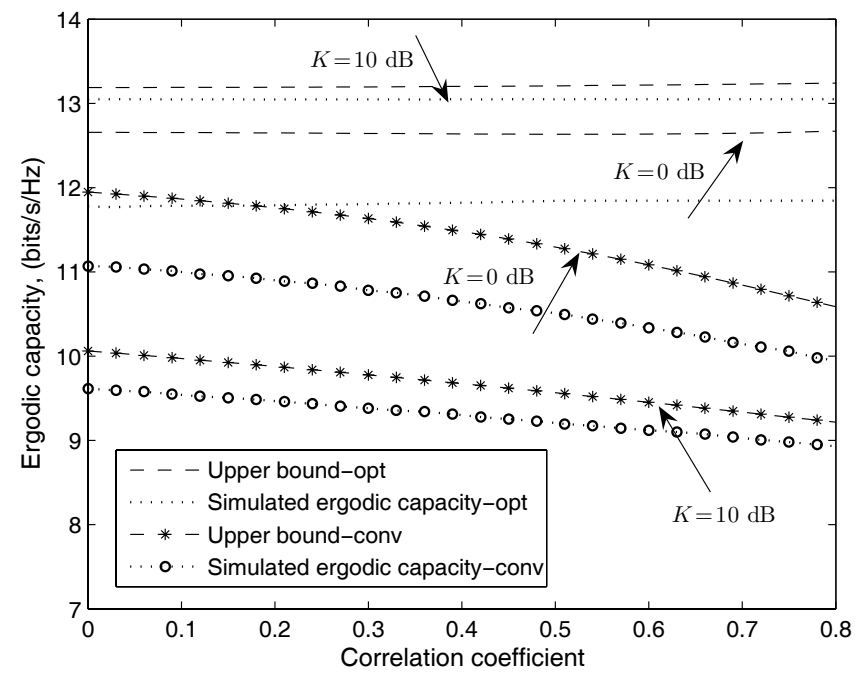

Fig. 5. Upper bound and simulated ergodic capacity as a function of the correlation coefficients $\delta_{R}=\delta_{T}(\rho=20 \mathrm{~dB})$.

correlation gets higher while the tightness of the corresponding bound is relatively improved in the high-correlation regime. This outcome is in agreement with [4], [10], [12].

At the last stage of the evaluation process, we consider the high-SNR deviation between the ergodic capacity and the proposed upper bound using the closed-form formulae in (27), (30) and (35). In Fig. 6, these analytical curves are overlaid with the outputs of a Monte-Carlo simulator with the match being remarkably good. The error associated with optimized configurations is systematically lower than that of conventional ones, as a result of the rank-deficiency of the former. What is more, it appears that the latter error has a much smaller dynamic range revealing that a high $K$-factor does not have an extensive impact on its value. On the other hand, the bound for the optimized configuration yields an enhanced tightness as $K$ increases and under strong Ricean conditions the corresponding offset is minimized. 


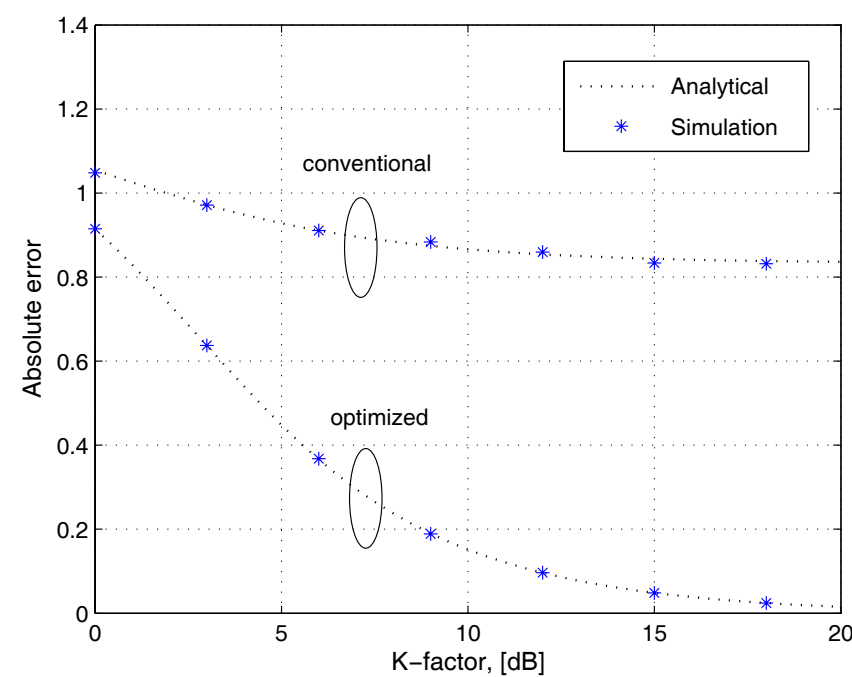

Fig. 6. Analytical and simulated absolute error of the upper bound in the high-SNR regime as a function of the $K$-factor $\left(\delta_{R}=0.2\right.$ and $\left.\delta_{T}=0.5\right)$.

\section{CONCLUSION}

An alternative simplified derivation of a tractable upper bound for the ergodic capacity of dual MIMO Ricean systems has been proposed, with the key concept originating from a widely used power normalization. The proposed bound depends merely on the SNR and the expected value of the determinant of either a non-central quadratic form or a noncentral Wishart matrix and, further, is not confined to the common case of rank-1 LoS components. The tightness of the bound was assessed in the low and high-SNR regions; in the former, the bound becomes asymptotically tight whereas in the latter the bound's offset tends to a constant value that was analytically determined and validated through MonteCarlo simulations. For the sake of completeness, we explored two different classes of LoS configurations, these were a conventional and an optimized architecture which benefits from the presence of strong deterministic components by offering two equal LoS eigenvalues. It was demonstrated that the bound is remarkably tight for the optimized case and marginally looser for conventional setups.

\section{APPENDIX A}

\section{PROOF OF THEOREM 1}

We begin by expressing the determinant of the quadratic form $\mathbf{Q} \sim \mathcal{C Q}_{2,2}\left(\mathbf{I}_{2}, \boldsymbol{\Sigma}, \boldsymbol{\Psi}, \mathbf{M}\right)$ as

$$
\begin{aligned}
E\left[\operatorname{det}(\mathbf{Q})^{v}\right] & =E\left[\operatorname{det}\left(\mathbf{X} \mathbf{X}^{H}\right)^{v}\right] \\
& =E\left[\operatorname{det}\left(\boldsymbol{\Sigma}^{1 / 2} \overline{\mathbf{X}} \overline{\mathbf{X}}^{H} \boldsymbol{\Sigma}^{1 / 2}\right)^{v}\right] \\
& =\operatorname{det}(\boldsymbol{\Sigma})^{v} E\left[\operatorname{det}\left(\overline{\mathbf{X}} \overline{\mathbf{X}}^{H}\right)^{v}\right]
\end{aligned}
$$

where $\overline{\mathbf{X}} \sim \mathcal{C N}_{2,2}\left(\overline{\mathbf{M}}, \mathbf{I}_{2} \otimes \mathbf{\Psi}\right)$. Using a result from [14] with the aid of the Cauchy-Binet formula, it can be shown that the matrix $\overline{\mathbf{X}}^{H} \overline{\mathbf{X}} \sim \mathcal{C W}_{2}(2, \mathbf{\Psi}, \boldsymbol{\Theta})$. The expectation of the determinant in (40) can now be evaluated through [13,
Theorem 1]

$$
\begin{aligned}
E\left[\operatorname{det}\left(\overline{\mathbf{X}} \overline{\mathbf{X}}^{H}\right)^{v}\right] & =\operatorname{det}(\boldsymbol{\Psi})^{v}\left[\frac{\tilde{\Gamma}_{2}(v+2)}{\tilde{\Gamma}_{2}(2)}\right] \\
& \times \operatorname{etr}(-\boldsymbol{\Theta})_{1} \widetilde{F}_{1}(v+2 ; 2 ; \boldsymbol{\Theta})
\end{aligned}
$$

where we have made use of the basic property, $\operatorname{det}(\mathbf{C D})=$ $\operatorname{det}(\mathbf{D C})$, for the determinant of the product of square matrices. The proof concludes after introducing the well-known Kummer relation for hypergeometric functions of one matrix argument, i.e. ${ }_{1} \widetilde{F}_{1}(a ; b ; \mathbf{S})=\operatorname{etr}(\mathbf{S})_{1} \widetilde{F}_{1}(b-a ; b ;-\mathbf{S})$.

\section{APPENDIX B \\ PROOF OF THEOREM 3}

The proof begins with the following key transformation

$$
E[\ln x]=\left.\frac{d}{d v} E\left[x^{v}\right]\right|_{v=0}
$$

which holds since, by definition, $x^{v}=e^{v \ln x}$. By combining (42) and (1) and denoting

$$
\zeta=E\left[\log _{2}(\operatorname{det}(\widetilde{\mathbf{W}}))\right]=\frac{1}{\ln 2} E[\ln (\operatorname{det}(\widetilde{\mathbf{W}}))]
$$

and

$$
\phi(v)=\left|(K+1)^{-1} \mathbf{R}_{r} \mathbf{R}_{t}\right|^{v}\left[\frac{\tilde{\Gamma}_{2}(v+2)}{\tilde{\Gamma}_{2}(2)}\right]{ }_{1} \widetilde{F}_{1}(-v ; 2 ;-\boldsymbol{\Theta})
$$

we can directly get

$$
\zeta=\left.\frac{1}{\ln 2} \frac{d}{d v} \phi(v)\right|_{v=0}=\left.\frac{1}{\ln 2} \frac{d}{d v} \ln \phi(v)\right|_{v=0}
$$

where the second equality follows since $\phi(0)=1$. It is easily seen that the above differentiation consists of three multiplicative terms. Treating each one separately due to the chain rule, the first term results in $\ln \left|\mathbf{R}_{r} \mathbf{R}_{t}\right|-2 \ln (K+1)$. The second one, may be rearranged to give

$$
\begin{aligned}
& \left.\frac{d}{d v}\left\{\ln \left(\frac{\tilde{\Gamma}_{2}(v+2)}{\tilde{\Gamma}_{2}(2)}\right)\right\}\right|_{v=0}=\left.\frac{d}{d v}\left\{\ln \left(\tilde{\Gamma}_{2}(v+2)\right)\right\}\right|_{v=0} \\
= & \left.\frac{d}{d v}\left\{\sum_{i=1}^{2} \ln (\Gamma(v+2-i+1))\right\}\right|_{v=0}
\end{aligned}
$$

which, through (31), yields $\psi(1)+\psi(2)$. Focusing now on the last term, we get (46) at the top of next page. The proof concludes after introducing a useful result from [13, Appendix II-B] for the nominator in (46) and simplifying the denominator using ${ }_{1} \widetilde{F}_{1}(\alpha ; \alpha ; x)=\exp (x)$. Substituting (45) and (46) into (44) and collecting common terms yields (30).

\section{REFERENCES}

[1] G. J. Foschini, "Layered space-time architecture for wireless communications in a fading environment when using multiple antennas," Bell Labs Tech. J., vol. 1, no. 2, pp. 41-59, Autumn 1996.

[2] I. E. Telatar, "Capacity of multi-antenna Gaussian channels," Europ. Trans. Telecommun., vol. 10, no. 6, pp. 585-595, Nov./Dec. 1999.

[3] A. Grant, "Rayleigh fading multi-antenna channels," EURASIP J. Appl. Signal Process., vol. 2002, no. 3, pp. 316-329, Mar. 2002.

[4] H. Shin and J. H. Lee, "Capacity of multi-antenna fading channels: spatial fading correlation, double scattering, and keyhole," IEEE Trans. Inf. Theory, vol. 49, no. 10, pp. 2636-2647, Oct. 2003. 


$$
\begin{aligned}
\left.\frac{d}{d v}\left\{\ln \left({ }_{1} \widetilde{F}_{1}(-v ; 2 ;-\boldsymbol{\Theta})\right)\right\}\right|_{v=0} & =\left.\frac{d}{d v}\left\{\ln \left(\operatorname{etr}(-\boldsymbol{\Theta})_{1} \widetilde{F}_{1}(v+2 ; 2 ; \boldsymbol{\Theta})\right)\right\}\right|_{v=0} \\
& =\left.\frac{d}{d v}\left\{\ln \left({ }_{1} \widetilde{F}_{1}(v+2 ; 2 ; \boldsymbol{\Theta})\right)\right\}\right|_{v=0} \\
& =\frac{\left.\frac{d}{d v}{ }_{1} \widetilde{F}_{1}(v+2 ; 2 ; \boldsymbol{\Theta})\right|_{v=0}}{{ }_{1} \widetilde{F}_{1}(2 ; 2 ; \boldsymbol{\Theta})}
\end{aligned}
$$

[5] Y.-H. Kim and A. Lapidoth, "On the log determinant of non-central Wishart matrices," in Proc. IEEE Int. Symp. Inform. Theory (ISIT), June 2003, p. 54.

[6] S. Jin and X. Gao, "Tight lower bounds on the ergodic capacity of Ricean fading MIMO channels," in Proc. IEEE Int. Conf. Commun. (ICC), May 2005, vol. 4, pp. 2412-2416.

[7] —, "Tight upper bound on the ergodic capacity of the Rician fading MIMO channels," in Proc. IEEE Wireless Commun. Networking Conf. (WCNC), Mar. 2005, vol. 1, pp. 402-407.

[8] M. R. McKay and I. B. Collings, "On the capacity of frequencyflat and frequency-selective Rician MIMO channels with single-sided correlation," IEEE Trans. Wireless Commun., vol. 5, no. 8, pp. 20382043, Aug. 2005.

[9] X. W. Cui, Q. T. Zhang, and Z. M. Feng, "Generic procedure for tightly bounding the capacity of MIMO correlated Rician fading channels," IEEE Trans. Commun., vol. 53, no. 5, pp. 890-898, May 2005.

[10] S. Jin, X. Gao, and X. You, "On the ergodic capacity of rank-1 Riceanfading MIMO channels," IEEE Trans. Inf. Theory, vol. 53, no. 2, pp. 502-517, Feb. 2007.

[11] M. R. McKay and I. B. Collings, "Improved general lower bound for spatially-correlated Rician MIMO capacity," IEEE Commun. Lett., vol. 10, no. 3, pp. 162-164, Mar. 2006.

[12] J. Salo, F. Mikas, and P. Vainikainen, "An upper bound on the ergodic mutual information in Rician fading MIMO channels," IEEE Trans. Wireless Commun., vol. 5, no. 6, pp. 1415-1421, June 2006.
[13] M. R. McKay and I. B. Collings, "General capacity bounds for spatially correlated Rician MIMO channels," IEEE Trans. Inf. Theory, vol. 51, no. 9, pp. 3121-3145, Sep. 2005.

[14] M. R. McKay, P. J. Smith, and I. B. Collings, "New properties of complex noncentral quadratic forms and bounds on MIMO mutual information," in Proc. IEEE Int. Symp. Inform. Theory (ISIT), July 2006, pp. 1209-1213.

[15] F. Bøhagen, P. Orten, G. E. Øien, and S. de la Kethulle de Ryhove, "Exact capacity expressions for dual-branch Ricean MIMO systems," IEEE Trans. Commun., vol. 56, no. 12, pp. 2214-2222, Dec. 2008.

[16] I. Sarris and A. R. Nix, "Design and performance assessment of high-capacity MIMO architectures in the presence of a line-of-sight component," IEEE Trans. Veh. Technol., vol. 56, no. 4, pp. 2194-2202, July 2007.

[17] M. Matthaiou, D. I. Laurenson, and C.-X. Wang, "Capacity study of vehicle-to-roadside MIMO channels with a line-of-sight component," in Proc. IEEE Wireless Commun. Networking Conf. (WCNC), Mar. 2008, pp. $775-779$.

[18] Ö. Oyman, R. Nabar, H. Bölcskei, and A. Paulraj, "Characterizing the statistical properties of mutual information in MIMO channels," IEEE Trans. Signal Process., vol. 51, no. 11, pp. 2782-2795, Nov. 2003.

[19] M. Abramowitz and I. A. Stegun, Handbook of Mathematical Functions With Formulas, Graphs, and Mathematical Tables. New York: Dover, 1972. 